\title{
Spontaneous Renal Artery Dissection Diagnosed by Unenhanced Magnetic Resonance Angiography: Case Report
}

\author{
Emanuele Casciani ${ }^{a} \quad$ Elisabetta Polettini ${ }^{a} \quad$ Gabriele Masselli $^{a} \quad$ Giovanni Stirati ${ }^{b}$ \\ Gianfranco Gualdi ${ }^{\mathrm{a}}$ \\ ${ }^{a}$ Radiology, Emergency Department, and b Nephrology, University of Rome 'La Sapienza', Rome, Italy
}

\section{Key Words}

Magnetic resonance imaging $\cdot$ Renal artery $\cdot$ Dissection

\begin{abstract}
A 47-year-old patient is presented who was admitted to the emergency department with complaints of right-sided flank pain and hypertension. His creatinine and glomerular filtration rate were $2.5 \mathrm{mg} / \mathrm{dl}$ and $37 \mathrm{ml} / \mathrm{min}$ respectively, so that contrast media administration was contraindicated. The unenhanced magnetic resonance angiography image obtained with 3D in-flow inversion recovery sequence showed right renal artery dissection, without aortic dissection. Selective renal angiography confirmed the diagnosis and a stent was placed in the renal artery. The patient was free of pain after stenting, with normalized laboratory values and blood pressure.

Copyright $\odot 2012$ S. Karger AG, Basel
\end{abstract}

\section{Introduction}

Spontaneous renal artery dissection (SRAD) is a rare occurrence with fewer than 200 cases reported in the literature. Due to its rarity, SRAD may be difficult to diag- nose and treat [1]. Abdomen multislice CT angiography is appropriate as the emergency non-invasive examination in the evaluation of patients with normal renal function or mild dysfunction [2]. Magnetic resonance angiography (MRA) is an alternative tool for renal arteries disease [3]. Until recently, gadolinium was considered safe in patients with renal dysfunction, however the recognition of the gadolinium-induced nephrogenic system fibrosis substantially limited the use of contrast-enhanced magnetic resonance examination in patients with a glomerular filtration rate of $<60 \mathrm{ml} / \mathrm{min}$ [4]; non-contrast-enhanced examinations are desirable in these patients. To our knowledge, there is no report of SRAD diagnosed by unenhanced MRA.

\section{Case Presentation}

We present a 47-year-old patient admitted to the emergency department with complaints of severe right-sided flank pain and blood pressure 145/95 mm Hg; this was the first detection of elevated pressure. Physical examination was unremarkable. He was tachycardic with a regular rhythm and clear breath sounds. His abdomen was soft, non-tender, and non-distended with normal bowel sounds, and he had no costovertebral tenderness bilaterally. He denied gross hematuria. The patient denied any past medical history, surgical history, and allergies.

\section{KARGER}

Fax +4161306 1234

E-Mail karger@karger.ch

www.karger.com (c) 2012 S. Karger AG, Basel

0042-1138/12/0894-0486\$38.00/0

Accessible online at:

www.karger.com/uin
Emanuele Casciani

Viale del Policlinico 155

IT-00161 Rome (Italy)

Tel. +3966638066

E-Mailemanuelecasciani@gmail.com 
Fig. 1. a The DWI (b value $=1,000)$ sequence shows a cortical and medullary wedge-shaped high-intensity signal area of the right kidney upper pole (arrows) according to renal infarction. b The transverse multiplanar reformatted view of the unenhanced MRA performed with a 3D in-flow inversion recovery sequence shows right renal artery dissection, beginning $2.5 \mathrm{~cm}$ after the ostium and extending to the hilum with intimal flap (curved arrow), true lumina (white arrow) and partial thrombosis of the false lumina (empty arrow).

Fig. 2. a Selective renal angiography confirms right renal artery dissection prior to stent placement. b Final angiography after stent delivery demonstrates normal caliber of the right renal artery.
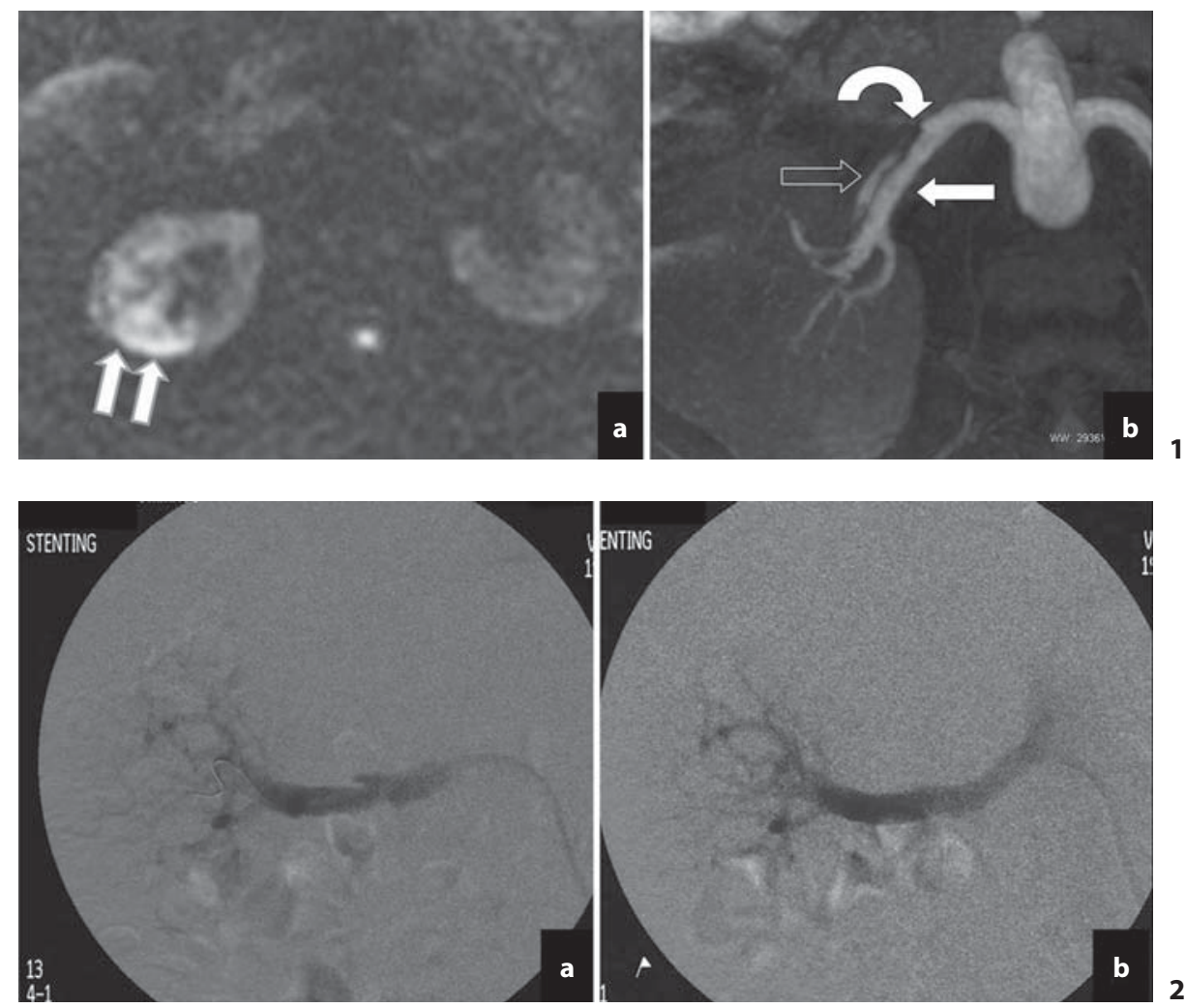

His creatinine and glomerular filtration rate were $2.5 \mathrm{mg} / \mathrm{dl}$ and $37 \mathrm{ml} / \mathrm{min}$ respectively, so that iodinated and gadolinium contrast media administration were contraindicated. Ultrasound of the abdomen, Doppler of the right artery, and CT of the abdomen without intravenous contrast media showed unremarkable findings. Since his pain was improved in the following hours, an MRI without intravenous contrast media was requested for further evaluation. The diffusion-weighted image (DWI) revealed a cortical and medullary wedge-shaped high-intensity signal area in the upper pole of the right kidney, consistent with renal infarction (fig. 1a). Therefore, unenhanced MRA imaging was performed with a $3 \mathrm{D}$ in-flow inversion recovery sequence completed with a transverse multiplanar reformatted view for vascular evaluation (fig. 1b). The sequence showed right renal artery dissection, beginning $2.5 \mathrm{~cm}$ after the ostium and extending to the hilum. There was no aortic dissection. In order to preserve renal function, a selective renal angiography was performed after adequate intravenous rehydration. Angiography was performed according to standard techniques for selective angiography via a 6-Fr coronary diagnostic catheter (Judkins right 4; Cordis, Miami Lakes, Fla., USA). Injection of $10 \mathrm{ml}$ of non-ionic iodinated contrast medium (320 mg/ml iodine, Visipaque; GE Healthcare, Oslo, Norway) was performed by hand (with a $10-\mathrm{ml}$ syringe) at a rate of about $5 \mathrm{ml} / \mathrm{s}$.

The examination confirmed the MRA diagnosis (fig. 2a) and a stent (Palmaz balloon-expandable stent; Cordis Endovascular, Miami, Fla., USA) was placed in the renal artery (fig. 2b). The patient was free of pain after stenting, with normalized laboratory values and blood pressure.

MRA of Spontaneous Renal Artery Dissection

\section{Discussion}

The etiology of SRAD is often unknown. A few factors have been identified as risk factors for SRAD including atherosclerosis, fibromuscular dysplasia, connective tissue disease, and other conditions that might affect the integrity of the renal artery, such as Marfan syndrome, Ehlers-Danlos syndrome, subadventitial angioma, and cystic medial necrosis. SRAD is more common among males (4:1 male:female ratio), smokers and usually observed in the fourth to sixth decade of life [2].

The most common symptoms of the SRAD are severe pain occurring unilaterally in the upper abdomen or flank and hypertension. The initial presentation of the SRAD can be non-specific with symptoms suggestive of renal colic. Because this condition is rare and the symptoms are vague, the diagnosis is often not considered until additional imaging studies are performed. A number of imaging modalities, such as multislice CT angiography scan, or MRI may be useful in diagnosis, but the gold standard is angiography, because it can precisely demonstrate the extent of the vascular involvement while identifying potential treatment options $[1,2,5]$. However, in patients who have poor renal function, as in our patient, 
who are pregnant, or who have inadequate intravenous access, the use of iodinate and/or gadolinium-based contrast material may be contraindicated or not feasible. In these patients, MRA techniques that do not require contrast material but are of equal quality and reliability as contrast-enhanced MRA techniques, would be desirable [6]. Only 1 reported patient with suspected SRAD has been previously studied with contrast-enhanced MRI [2]. In that case report, MRI identified renal infarction, but not the renal artery dissection [2].

Our case report showed the MRI possibility to identify renal infarction by using DWI sequence, and SRAD by using a 3D in-flow inversion recovery sequence without contrast media administration; this is particularly useful in patients with renal failure.

\section{Conclusion}

Isolated SRAD is rare and often presents as a diagnostic and therapeutic challenge. Unenhanced MRI and MRA are helpful in making the diagnosis of SRAD complicated with renal infarction in acute patients with renal failure.

\section{References}

1 Muller BT, Reiher L, Pfeiffer T, et al: Surgical treatment of renal artery dissection in $25 \mathrm{pa}$ tients: indications and results. J Vasc Surg 2003;37:761-768.

-2 Kanofsky JA, Lepor H. MD: Spontaneous renal artery dissection. Rev Urol 2007;9:156160 .
Leiner T, Michaely H: Advances in contrastenhanced MR angiography of the renal arteries. Magn Reson Imaging Clin N Am 2008;16:561-572.

4 Martin DR, Semelka RC, Chapman A, et al: Nephrogenic systemic fibrosis versus contrast-induced nephropathy: risks and benefits of contrast-enhanced MR and CT in renally impaired patients. J Magn Reson Imaging 2009;30:1350-1356.
5 Mudrick D, Arepally A, Geschwind JF: Spontaneous renal artery dissection: treatment with coil embolization. J Vasc Interv Radiol 2003;14:497-500.

6 6 Stafford RB, Sabati M, Haakstad MJ, Mahallati H, Frayne R: Unenhanced MR angiography of the renal arteries with balanced steady-state free precession Dixon method. AJR Am J Roentgenol 2008;191:243-246. 